\title{
Electronic properties of iron and cobalt impurity centres in germanium
}

\author{
J. Lauwaert ${ }^{1}$, J. Vanhellemont ${ }^{1}$, E. Simoen ${ }^{2}$, H. Vrielinck ${ }^{1}$ and P. Clauws ${ }^{1}$ \\ ${ }^{1}$ Department of Solid State Sciences, Ghent University, Krijgslaan 281-S1, 9000 Gent, Belgium \\ ${ }^{2}$ IMEC, Kapeldreef 75, 3001 Leuven, Belgium
}

\begin{abstract}
The electronic properties of the $3 d$ transition metal impurities iron and cobalt in crystalline germanium have been investigated by means of deep level transient spectroscopy. The metals were implanted at $90 \mathrm{keV}$ and diffused deeper into the bulk during a thermal anneal at $500^{\circ} \mathrm{C}$, which yielded spectra specific for the implanted metal. It was found that Fe introduces one deep electron trap and one deep hole trap. For Co one deep electron trap and two hole traps were observed. The capture cross-section of both electron and hole traps has been directly measured. The results are in agreement with the metals being present on substitutional lattice sites forming multiple acceptor states. It is concluded that Fe and Co in germanium are double acceptors, with in the case of Co an additional donor level close to the valence band.
\end{abstract}




\section{Introduction}

The electronic properties of metal impurities in germanium have already been studied in the 1950's using resistivity/Hall effect and photo-electric measurements. Summaries of results with regard to energy levels and carrier capture cross sections for several metals obtained in those early studies, may be found in Refs.1-4. A general picture that emerges is that transition metals in germanium predominantly form multiple-acceptor centres corresponding with several deep levels in the band gap. According to a simple valence bond model this observation is in agreement with a preferential occurrence of the impurities on substitutional sites $^{5}$, in marked contrast with silicon where metal impurities have been found to prefer interstitial sites ${ }^{6}$.

Due to the recent perspective to apply germanium in advanced electronic devices, a renewed interest in the electronic properties of impurities and defects in germanium has emerged in the last decade. Since the early studies mentioned above, deep level transient spectroscopy (DLTS) has become available as the preferential technique to study deep level centres in semiconductors. Besides the higher versatility and better energy resolution of DLTS compared with measurements of resistivity and Hall-coefficient as a function of temperature, the method is also more suitable to investigate samples with deep levels in a thin layer below the surface, as in the case of impurity in-diffused samples. DLTS of metal in-diffused germanium has been very successful in the case of $\mathrm{Cu}, \mathrm{Ag}, \mathrm{Au}$ and $\mathrm{Ni}^{7}$. For other transition metals however, the assignment of levels observed with DLTS of metal in-diffused germanium samples is less well established. A major difficulty with in-diffusion in germanium is that DLTS-bands due to rapidly diffusing contaminants like $\mathrm{Cu}$ or $\mathrm{Ni}$ often dominate the spectrum, obscuring features from the intended impurity. Similar difficulties were e.g. encountered in diffusivity studies of $\mathrm{Co}^{8}$. For the DLTS study in the present paper, 
a different approach has been used, i.e. ion implantation followed by a thermal anneal to restore the implantation damage and diffuse the impurities deeper into the sample. Our investigations of the last few years have shown that this method to dope germanium with transition metals is more adequate than diffusion from an intentionally contaminated surface, yielding distinct results for different metals without interference of other contaminants ${ }^{9-11}$.

In this paper we present DLTS results concerning the $3 d$ transition metals Fe and Co in germanium. In earlier work, resistivity and Hall-effect measurements of germanium crystals that had been doped with $\mathrm{Fe}$ in the melt have revealed the presence of levels at $E_{C}-0.27 \mathrm{eV}$ and $E_{V}+0.34 \mathrm{eV}^{12,13}\left(E_{C}\right.$ and $E_{V}$ are the energy of the conduction and valence band edge respectively). No evidence was obtained with regard to the donor or acceptor character of the Fe-levels; these Fe-related levels were however assumed to belong to a double acceptor, in analogy with the case of $\mathrm{Au}$ in germanium ${ }^{12}$. Electrical measurements of germanium meltdoped with Co showed the presence of levels at $E_{C}-0.31 \mathrm{eV}$ and $E_{V}+0.25 \mathrm{eV}$. Based on a careful study of the compensation behaviour of the Co dopant they were assigned to acceptors 14. Later the occurrence of a third level at $E_{V}+0.09 \mathrm{eV}$ due to Co and assigned to a deep donor was reported by Tyler ${ }^{1}$ without further detail. The presence of a deep donor level associated with Co was confirmed by Barnik et al ${ }^{15}\left(E_{V}+0.083 \mathrm{eV}\right)$; the latter level is in agreement with the occurence of a photo-ionisation edge at $0.083 \mathrm{eV}$ in Co-doped germanium 15

Here we present a systematic DLTS study of n- and p-type germanium wafers implanted with Fe or Co and thermally annealed, intended to obtain a complete level spectrum of the centres formed and to obtain precise deep level parameters, thus representing an elaborate extension of preliminary results ${ }^{10}$. 


\section{Experimental}

The starting material were n- and p-type single crystal germanium wafers (Umicore), with a Sb shallow donor concentration of $5 \times 10^{13} \mathrm{~cm}^{-3}$ for n-type and a Ga shallow acceptor concentration of $1 \times 10^{14} \mathrm{~cm}^{-3}$ for p-type. The wafers were implanted with $\mathrm{Fe}$ or Co at an energy of $90 \mathrm{keV}$; for each metal a wafer with a lower $\left(5 \times 10^{13} \mathrm{~cm}^{-2}\right)$ and a higher $\left(5 \times 10^{14} \mathrm{~cm}^{-}\right.$ ${ }^{2}$ ) implantation dose was prepared. A 5 min post-implantation thermal anneal was applied at $500^{\circ} \mathrm{C}$ in a $\mathrm{N}_{2}$ ambient in order to restore the implantation damage and to diffuse-in the metal impurities. Schottky diodes for DLTS were prepared by evaporation of a Au barrier (on ntype) or In barrier (on p-type). The evaporation was preceded by a short etch in a $\mathrm{HNO}_{3}: \mathrm{HF}$ (3:1) solution. Capacitance DLTS measurements were made with a Fourier Transform instrument (Phystech FT1030) equipped with a Boonton 72B capacitance meter with an AC test signal of $1 \mathrm{MHz}$. In addition to standard DLTS using voltage pulses, n-type samples were also measured with optical DLTS (ODLTS) where an optical pulse from a diode laser (980 nm wavelength) was applied. For the latter experiments, semi-transparent Au barriers were evaporated.

\section{DLTS results}

Before treating the DLTS results in detail, we want to remark that the spectra observed are essentially the same for the samples that received the lower or the higher implantation dose. Since diodes with a lower leakage current and spectra with a better signal-to-noise ratio were systematically obtained for samples that received the lower dose, the latter samples have been used preferentially for the detailed analysis. 


\section{A. Iron}

The DLTS spectrum of Fe-implanted germanium is shown in Fig.1a,b. The spectrum consists of two peaks from majority carrier traps: Fe-E1 observed in n-type corresponding with an electron trap and Fe-H1 observed in p-type corresponding with a hole trap. The amplitude of other features is negligible in comparison with these two main resonances.

At first we describe the characterisation of Fe-E1. The thermal emission rate from an electron trap may be written as ${ }^{16}$ :

$$
e_{n}(T)=\sigma_{n}(T) v_{n}(T) N_{c}(T) \exp \left(-\frac{\Delta G(T)}{k_{B} T}\right)
$$

where $\sigma_{n}(T)$ is the electron capture cross-section, $v_{n}(T)$ the thermal velocity of the electrons, $N_{c}(T)$ the effective density of states in the conduction band and $\Delta G(T)$ the Gibbs free energy of ionisation corresponding with the distance of the energy level to the conduction band. As will be shown below, the electron capture cross-section of Fe-E1 displays an activated temperature dependence in agreement with multiphonon-assisted capture ${ }^{17}$, which can be described by:

$$
\sigma_{n}(T)=\sigma_{\infty} \exp \left(-\frac{\Delta E_{\sigma}}{k_{B} T}\right)
$$

Expressing $\Delta G$ in terms of the changes in enthalpy $\Delta H$ and entropy $\Delta S$, Eq.(1) may also be written as ${ }^{16}$ :

$$
e_{n}(T)=\sigma_{\infty} v_{n}(T) N_{c}(T) \exp \left(\frac{\Delta S}{k}\right) \exp \left(-\frac{\Delta H+E_{\sigma}}{k T}\right)
$$

Taking account of the temperature dependence of $v_{n}$ and $N_{c}$, Eq.(3) may be presented in a form convenient for DLTS analysis: 


$$
e_{n}(T)=K_{T} T^{2} \exp \left(-\frac{E_{n a}}{k T}\right)
$$

In this equation the pre-exponential factor $K_{T}$ and the apparent activation energy $E_{n a}$ are the so-called signature of the deep level. The signature may be extracted from an Arrhenius diagram of the experimental emission rates. An alternative for $K_{T}$ and proportional to it, is the apparent capture cross-section $\sigma_{n a}{ }^{16}$; that is related to the true cross-section by:

$$
\sigma_{n a}=\sigma_{\infty} \exp \left(\frac{\Delta S}{k}\right)
$$

The Arrhenius diagram of Fe-E1, constructed from the recorded capacitance transients using different rate windows and Fourier coefficients or correlation functions ${ }^{18}$, is shown in Fig.2. The corresponding values of the signature and apparent capture cross-section are listed in Table 1.

The true capture cross-section of Fe-E1 has been directly measured by the variable pulse length method, i.e. from the dependence of the DLTS amplitude on the voltage pulse duration. The experimental data were fitted and a value for the capture cross-section extracted by a procedure taking account of slow capture and allowing for a defect concentration profile 19. Repeating the measurement at different temperatures, an exponential dependence of the capture cross-section on temperature is found, which is represented in the Arrhenius diagram of Fig.3. The corresponding capture parameters of the level according to Eq.(2) are also listed in Table 1. The apparent activation energy may now be corrected by the capture barrier $\Delta E_{\sigma}=0.050 \mathrm{eV}$, yielding the enthalpy of the level: $\Delta H=E_{n a}-\Delta E_{\sigma}=0.292 \mathrm{eV}$. Finally, the entropy of the level may be calculated from the true and apparent capture cross-section using Eq.(5), which yields $\Delta S=2.0 k_{B}$. The relatively low value of the electron capture crosssection and its activated behaviour are in agreement with capture into a negative charge state, which may be the case if the defect is a multiple acceptor. 
The signature of $\mathrm{Fe}-\mathrm{H} 1,\left(K_{T}, E_{p a}\right)$ according to the analogue of Eq.(4) for holes, as well as the apparent capture cross-section $\sigma_{p a}$, are listed in the lower part of Table 1; the Arrhenius diagram of Fe-H1 is also shown in Fig.2. Rather surprisingly for an assumed acceptor level, the true hole capture cross-section of Fe-H1 could be measured using very short filling pulses, as demonstrated in the plot of Fig.4. No temperature dependence could be observed within the measurement range of the peak of 130-145 K; the value of the constant cross-section is indicated as $\beta$ in Table 1 . Accordingly the enthalpy value of Fe-H1 in Table 1 is identical to the apparent activation energy, while the entropy value was calculated in analogy with Eq.(5). No distinct shift of Fe-H1 indicating the occurrence of field-enhanced hole emission has been observed.

The concentration of the Fe-E1 and Fe-H1 traps as a function of distance from the barrier has been measured using DLTS with constant pulse height and varying bias. In both nan p-type a diffusion-like profile is found, very similar to the profiles of implanted $\mathrm{Ti}$ published in Ref.11. Here again the concentration profiles of Fe-E1 and Fe-H1 are in very good agreement within the accuracy of the procedure, supporting the assumption that the levels belong to the same Fe-related defect. The deep level concentration in the measurement range is below $10 \%$ of the background concentration, justifying the use of DLTS formulae valid at low relative concentrations.

Fig.1c shows the ODLTS result of Fe-implanted n-type germanium. The optical injection of electrons and holes into the depletion layer leads to the observation of not only Fe-E1 but also of the minority carrier (hole) trap Fe-H1 in the same spectrum. This result demonstrates that both traps occur simultaneously in the n-type samples, which further supports the assignment to the same defect. 


\section{B. Cobalt}

A typical DLTS spectrum of the majority carrier traps in Co-implanted germanium is displayed in Fig.5a,b. In n-type a single band labeled Co-E1 is observed, while in p-type two bands $\mathrm{Co}-\mathrm{H} 1$ and $\mathrm{Co}-\mathrm{H} 2$ are present with the same amplitude. Again the other features in the spectrum are negligible in comparison with the three main resonances. The Arrhenius diagram of Co-E1 according to Eq.(4) is shown in Fig.6a, the signature and apparent capture cross section are listed in Table 1. As for Fe-E1, the true electron capture cross section could be directly measured and was found to be thermally activated in agreement with Eq.(2). The activated dependence is displayed in Fig.7 and the capture parameters included in Table 1. The capture barrier amounts to $\Delta E_{\sigma}=0.010 \mathrm{eV}$, allowing to correct the apparent activation energy of the level to an enthalpy of $\Delta H=0.315 \mathrm{eV}$ and resulting in an entropy value of $\Delta S=3.9 k_{B}$. The capture data are again in agreement with a multiple acceptor.

The Arrhenius diagrams of Co-H2 and Co-H1 are shown in Fig.6a,b and the signatures have also been included in Table 2. Again as in the case of Fe, we were able to measure the true capture cross sections of Co-H1 and Co-H2 using very short filling pulses. In the present case however the cross sections showed a weak temperature dependence which could be fitted according to ${ }^{20}$ :

$$
\sigma_{p}(T)=\frac{\alpha}{T}+\beta
$$

as shown in Fig.8a,b; the fitting parameters are included in Table 1. Taking account of the temperature dependence of $\sigma_{p}$, the apparent activation energy $E_{p a}$ can be corrected to yield the values for $\Delta H$ appearing in the lower part of Table 1, where also the entropy values have been included. Again no distinct shift of the position of the bands $\mathrm{Co}-\mathrm{H} 1$ or $\mathrm{Co}-\mathrm{H} 2$ with electric field has been observed. 
The concentration of Co-E1 in n-type and of Co-H1 and Co-H2 in p-type displays similar diffusion-like profiles, indicating that the levels most probably belong to the same defect. This is substantiated by the ODLTS result of n-type displayed in Fig.5c, where besides Co-E1, both Co-H1 and Co-H2 appear as minority carrier traps.

\section{Discussion}

All observations indicate that the spectra displayed in Figs. 1 and 5 are uniquely related to the implanted metals $\mathrm{Fe}$ and Co, respectively. In a preliminary study where Ge-implanted wafers were used as a reference ${ }^{9}$, DLTS bands due to the implantation damage are different from the bands presented here and these damage related bands are also removed by a $500^{\circ} \mathrm{C}$ anneal. Moreover, the present spectra are essentially the same for low and high dose implants with the same metal, i.e they display the same DLTS bands. The spectra are distinctly different between $\mathrm{Fe}$ and $\mathrm{Co}$ and are also different from the spectra due to other transition metals, i.e. Ti and $\mathrm{Cr}$ implanted ${ }^{11}$ or $\mathrm{Ni}$ and $\mathrm{Cu}$ diffused ${ }^{7}$.

The Fe-E1 and Fe-H1 bands most probably belong to the same Fe-related defect just as the Co-E1, Co-H1 and Co-H2 bands most probably belong to the same Co-related defect. These assignments are supported by the similar concentration profile of corresponding bands and in the case of cobalt also by the almost identical amplitude of Co-H1 and Co-H2 in Fig.5b. Another strong indication is the simultaneous occurrence of Fe-E1 and Fe-H1 in the same n-type sample and similarly for Co-E1 and Co-H1/H2, as shown in the ODLTS spectra in Fig.1c and Fig.5c.

The data presented here are in very good agreement with the results from the early electrical measurements ${ }^{12-15}$ : for Fe as well as for Co the same number of levels with the same position in the band gap are found. If we compare the $\Delta H$ values in Table 1 with the 
level energies from the electrical studies mentioned in the introduction, we find a fair agreement for Fe-E1 and an excellent agreement for the other four levels. Independently of the doping method, i.e doping in the melt vs. implantation, and characterization technique, the same levels have been observed and the levels are therefore uniquely related to the respective metal dopant.

The latter is substantiated by the observation of the Fe-E1 and Co-E1 levels in DLTS of Fe- and Co-germanide Schottky barriers on n-type germanium, interpreted as a result of indiffusion of the respective metal from the germanide barrier into the germanium substrate at elevated germanide formation temperatures ${ }^{21,22}$; a minority band appearing upon application of forward voltage pulses probably corresponds with level Co-H2. Recently, a DLTS study of Fe-implanted n-type germanium has been published ${ }^{23}$, in which a majority band similar to Fe-E1 was reported as well as a minority band similar to $\mathrm{Fe}-\mathrm{H} 1$ applying optical injection. The signatures in Ref.23 deviate however rather significantly from the present results, especially in the case of the minority band. This may be a consequence of the presence of a shoulder on the low-temperature side of the majority band in Ref.23 which, according to the authors, is due to the presence of hydrogen as a result of the etching procedure used. This shoulder is absent in the spectrum of Fig.1a. In addition the signature of the minority band was obtained in possibly complex injection conditions which is less straightforward than in the case of the majority Fe-H1 band in Fig.1b.

The electron capture cross section measured for the levels Fe-E1 and Co-E1 is thermally activated. This is an indication of multiphonon-assisted capture against a repulsive barrier, as in the case of electron capture into a negative charge state of the defect ${ }^{3,17}$. Similar observations were made for the electron traps associated with the substitutional metal impurities $\mathrm{Ti}, \mathrm{Cr}, \mathrm{Cu}, \mathrm{Ag}$ and $\mathrm{Au}$ in germanium ${ }^{7,11}$. The latter traps correspond with the -/2or 2-/3- level of multiple acceptors, so that electron capture at these levels occurs into the 
singly or doubly negative charge state. Similarly, the electron capture data for Fe-E1 and CoE1 are an indication of at least double acceptors, which is in agreement with the conclusion from the earlier electrical measurements ${ }^{12,14}$. In addition, the value of $\Delta E_{\sigma}=0.050 \mathrm{eV}$ for $\mathrm{Fe}-$ $\mathrm{E} 1$ is in perfect agreement with the value of $0.05 \mathrm{eV}$ for the activation energy of the capture cross section $\sigma_{\mathrm{n}}^{-}$of Fe in germanium as determined by the photoconductive decay method ${ }^{23}$. This correspondence substantiates the assignment of Fe-E1 to the double acceptor level $\mathrm{Fe}^{-/ 2-}$. The value of the electron capture cross section of Fe-E1 extrapolated to $300 \mathrm{~K}$ of $5 \times 10^{-16} \mathrm{~cm}^{2}$ is also in good agreement with the value of $\sigma_{\mathrm{n}}^{-}$slightly above $10^{-16} \mathrm{~cm}^{2}$ reported in Ref.24.

The assignment to double acceptors implies that Fe-H1 and Co-H2 belong to the levels $\mathrm{Fe}^{0 /-}$ and $\mathrm{Co}^{0 /-}$ respectively. Hole capture at similar levels occurs into the singly negative charge state, i.e. into a state attractive for holes. It was therefore not readily expected that the capture would be measurable by the variable pulse length method at the shallow dopant concentrations of the samples used for the present study. E.g. in the case of $\mathrm{Ti}$ and $\mathrm{Cr}$, where similar wafer material was used for the implantation, hole capture occurred too fast to be measured ${ }^{11}$. Another remark with respect to the assumed acceptor nature of Fe-H1 and Co$\mathrm{H} 2$ is the absence in our experiments of a distinct field-enhanced hole emission. In Ref.23 a shift of the minority band belonging to Fe was reported, in agreement with the Poole-Frenkel effect. Extrapolation to zero field resulted however in a value of $\Delta H=0.37 \mathrm{eV}^{22}$, rather different from the present value for $\mathrm{Fe}-\mathrm{H} 1$ of $0.345 \mathrm{eV}$ and the level at $E_{V}+0.34 \mathrm{eV}$ from the electrical measurements ${ }^{12,13}$. This discrepancy throws some doubt on the measurement under injection conditions or on the identity of the minority carrier band observed in Ref.23. The spectra of $\mathrm{Fe}-\mathrm{H} 1$ and $\mathrm{Co}-\mathrm{H} 2$ in the present study have been measured as majority carrier bands in diodes with normal characteristics and well defined electric field values in the range where the emission occurs. We therefore have no reason to doubt about the absence of a Poole-Frenkel like shift in our measurements. Again the observations are different from the 
case of $\mathrm{Ti}$ and $\mathrm{Cr}$ implanted germanium, where the acceptor levels in the lower half of the band gap do display Poole-Frenkel shifts ${ }^{11}$. The fact that Fe and Co have been implanted in the same batch of germanium wafers and measured in similar diodes and circumstances as $\mathrm{Ti}$ and $\mathrm{Cr}$, strengthens our belief that a Poole-Frenkel shift would have been observed if present.

The slower hole capture and absence of field enhanced hole emission may question the assignment of the Fe-H1 and $\mathrm{Co}-\mathrm{H} 2$ traps to acceptor states. At least for $\mathrm{Co}-\mathrm{H} 2$ however the acceptor nature was established through the observed compensation behavior ${ }^{14}$. Moreover as discussed above, the activated electron capture of Fe-E1 and Co-E1 indicates that we are concerned with at least double acceptors. It may be remarked that $\mathrm{Fe}-\mathrm{H} 1$ and $\mathrm{Co}-\mathrm{H} 2$ correspond with levels that are situated much deeper in the band gap (at greater distance from the valence band edge) than the levels $\mathrm{Ti}-\mathrm{H} 1\left(E_{V}+0.028 \mathrm{eV}\right)$ and $\mathrm{Cr}-\mathrm{H} 2 / \mathrm{H} 3\left(E_{V}+\right.$ 0.048/0.095 eV), which in comparison may be considered semi-shallow acceptors. This different energy position and possible corresponding difference in electrical potential around the defects may explain the deviation of $\mathrm{Fe}-\mathrm{H} 1$ and $\mathrm{Co}-\mathrm{H} 2$ from the typical hole capture and emission behaviour of acceptor states.

\section{Conclusion}

In this paper the energy levels of $\mathrm{Fe}$ and Co impurities in germanium implanted with the respective metals have been investigated using DLTS. The results are in very good agreement with earlier published results from electrical measurements using melt-doped

germanium crystals ${ }^{12-15}$, so that the levels and their assignment to $\mathrm{Fe}$ and Co may be considered as well established. The levels belong to one Fe and one Co related defect, respectively. The activated capture cross section of Fe-E1 and Co-E1 confirms the multiple acceptor nature of the respective defects. In the case of Fe, introducing two levels, the earlier 
assumption of a double acceptor defect ${ }^{12}$ is accordingly confirmed. For Co three levels are observed, with Co-H1 according to compensation studies corresponding with a very deep donor state ${ }^{1,15}$; the other two levels may accordingly be assigned to double acceptor states as well. The charge states corresponding with the observed energy levels are listed in the last column of Table 1. The lower than expected hole capture cross section and the absence of field enhanced hole emission for the acceptor levels $\mathrm{Fe}^{0 /-}$ and $\mathrm{Co}^{0 /-}$ may be related to their deep energy position in the band gap.

The double acceptor nature of the defects is indicative of a substitutional position of the $\mathrm{Fe}$ and Co impurities in germanium and may be qualitatively explained by a tetrahedral bonding model ${ }^{1}$. In this respect $\mathrm{Fe}$ and $\mathrm{Co}$ are similar to $\mathrm{Ti}$ and $\mathrm{Ni}$ which also have a $4 s^{2}$ outer shell configuration and give rise to double acceptors ${ }^{11,25}$. It is remarkable that for all transition metals studied so far the multiple acceptor nature is in agreement with a valence bond model for substitutional impurities taking account of the $4 s$ occupation of the atom, i.e. double acceptors in the case of $\mathrm{Ti}, \mathrm{Fe}, \mathrm{Co}$ and $\mathrm{Ni}$ and triple acceptors in the case of $\mathrm{Cr}$ and $\mathrm{Cu}$ $7,11,25$

\section{Acknowledgments}

Part of this work has been made in cooperation with Umicore and with the support of the Institute for the Promotion of Science and Technology (IWT) and the Research Foundation-Flanders (FWO-Vlaanderen). 


\section{References}

1. W.W. Tyler, J. Phys. Chem. Solids 8, 59 (1959)

2. S.M. Sze and J.C. Irvin, Solid-State Electron. 11, 599 (1968)

3. A.G. Milnes, Deep Impurities in Semiconductors (Wiley, New York, 1973)

4. E. Simoen and C. Claeys, Germanium Based Technologies: From Materials to Devices, edited by C. Claeys and E. Simoen (Elsevier, Amsterdam, 2007)

5. R.C. Newman and W.W. Tyler, Solid State Phys. 8, 49 (1959)

6. E.R. Weber, Appl. Phys. A: Solids Surf. 30, 1 (1983)

7. P. Clauws and E. Simoen, Mater. Sci. Semicond. Process. 9, 546 (2006)

8. L. Lerner and N.A. Stolwijk, Appl. Phys. Lett. 93, 032107 (2008)

9. S. Forment, J. Vanhellemont, P. Clauws, J. Van Steenbergen, S. Sioncke, M. Meuris, E. Simoen and A. Theuwis, Mater. Sci. Semicond. Process. 9, 559 (2006)

10. P. Clauws, J. Van Gheluwe, J. Lauwaert, E. Simoen, J. Vanhellemont, M. Meuris and A. Theuwis, Physica B 401-402, 188 (2007)

11. J. Lauwaert, J. Van Gheluwe, J. Vanhellemont, E. Simoen and P. Clauws, J. Appl. Phys. 105, 073707 (2009)

12. W.W. Tyler and H.H. Woodbury, Phys. Rev. 96, 874 (1954)

13. K. D. Glinchuk, E.G. Miseliuk and N.N. Fortunatova, Sov. Phys. - Techn. Phys. 2, 2283 (1957)

14. W.W. Tyler, R. Newman and H.H. Woodbury, Phys. Rev. 97, 669 (1955)

15. M.I. Barnik, B.I. Beglov, D.A. Romanychev and Yu.S. Kharionovskii, Sov. Phys. Semicond. 5, 87 (1971)

16. P. Blood and J.W. Orton, The Electrical Characterization of Semiconductors: Majority Carriers and Electron States (Academic, New York, 1992)

17. C.H. Henry and D.V. Lang, Phys. Rev. B 15, 989 (1977) 
18. S. Weiss and R. Kassing, Solid-State Electron. 31, 1733 (1988)

19. J. Lauwaert, J. Van Gheluwe and P. Clauws, Rev. Sci. Instrum. 79, 093902 (2008)

20. J. Lauwaert and P. Clauws, Thin Solid Films 518, 2330 (2010)

21. E. Simoen, K. Opsomer, C. Claeys, K. Maex, C. Detavernier, R.L. Van Meirhaeghe and P. Clauws, Solid State Phenomena 131-133, 47 (2008)

22. E. Simoen, K. Opsomer, C. Claeys, K. Maex, C. Detavernier, R.L. Van Meirhaeghe and P. Clauws, J. Appl. Phys. 104, 023705 (2008)

23. Y. Gurimskaya, D. Mathiot, A. Sellai, P. Kruszewski, L. Dobaczewski, A. Nylandsted Larsen and A. Mesli, J. Appl. Phys. 110, 113707 (2011)

24. A.D. Belyaev and V.G. Malogolovets, Soviet Phys. Solid State 5, 2229 (1964)

25. G. Huylebroeck, P. Clauws, E. Simoen and J. Vennik, Solid State Commun. 82, 367 (1992) 
Figure captions

Figure $1 \quad$ DLTS and ODLTS of Fe-implanted germanium. $\tau_{\text {window }}=222 \mathrm{~ms}$

Figure 2 Arrhenius diagrams of the traps Fe-E1 and Fe-H1

Figure 3 Temperature dependence of the electron capture cross section of level Fe-E1

Figure 4 Amplitude of the DLTS signal of Fe-H1 as a function of filling pulse length at $\mathrm{T}=135 \mathrm{~K}$; squares: experimental data; full line: fitted dependence

Figure 5 DLTS and ODLTS of Co-implanted germanium. $\tau_{\text {window }}=222 \mathrm{~ms}$

Figure 6 Arrhenius diagrams of the traps Co-E1, Co-H1 and Co-H2

Figure $7 \quad$ Temperature dependence of the electron capture cross section of level Co-E1

Figure 8 Temperature dependence of the hole capture cross section of levels Co-H1 and $\mathrm{Co}-\mathrm{H} 2$ 


\begin{tabular}{|c|c|c|c|c|c|c|c|c|}
\hline Band & $E_{n a}$ & $K_{T}$ & $\sigma_{n a}$ & $\Delta E_{\sigma}$ & $\sigma_{\infty}$ & $\Delta H$ & $\Delta S$ & Level \\
& $\mathrm{eV}$ & $\mathrm{s}^{-1} \mathrm{~K}^{-2}$ & $\mathrm{~cm}^{2}$ & $\mathrm{eV}$ & $\mathrm{cm}^{2}$ & $\mathrm{eV}$ & $k_{B}$ & \\
\hline $\mathrm{Fe}-\mathrm{E} 1$ & 0.342 & $7.5 \times 10^{7}$ & $2.6 \times 10^{-14}$ & 0.050 & $3.7 \times 10^{-15}$ & 0.292 & 2.0 & $\mathrm{Fe}^{-/ 2-}$ \\
$\mathrm{Co}-\mathrm{E} 1$ & 0.325 & $3.3 \times 10^{8}$ & $1.1 \times 10^{-13}$ & 0.010 & $2.3 \times 10^{-15}$ & 0.315 & 3.9 & $\mathrm{Co}^{-/ 2-}$ \\
\hline Band & $E_{p a}$ & $K_{T}$ & $\sigma_{p a}$ & $\alpha$ & $\beta$ & $\Delta H$ & $\Delta S$ & $\mathrm{Level}^{-15}$ \\
\hline Fe-H1 & 0.345 & $2.0 \times 10^{9}$ & $2.1 \times 10^{-12}$ & 0 & $2.3 \times 10^{-14}$ & 0.345 & 4.3 & $\mathrm{Fe}^{0 /-}$ \\
Co-H1 & 0.086 & $6.8 \times 10^{8}$ & $7.2 \times 10^{-13}$ & $8.4 \times 10^{-13}$ & $1.9 \times 10^{-14}$ & 0.086 & 3.1 & $\mathrm{Co}^{+/ 0}$ \\
Co-H2 & 0.254 & $1.7 \times 10^{7}$ & $1.8 \times 10^{-14}$ & $7.4 \times 10^{-13}$ & $9.4 \times 10^{-16}$ & 0.261 & 1.4 & $\mathrm{Co}^{0 /-}$ \\
\hline
\end{tabular}

Table 1 Deep level parameters and assignment of the traps corresponding with Fe and Co impurities in germanium. 